\title{
A Survey on the Square Peg Problem
}

\section{Benjamin Matschke}

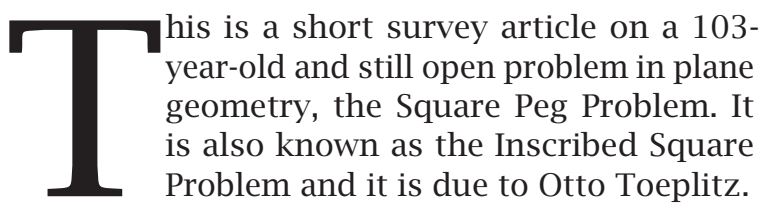

Conjecture 1 (Square Peg Problem, [39]). Every continuous simple closed curve in the plane $\gamma: S^{1} \rightarrow$ $\mathbb{R}^{2}$ contains four points that are the vertices of a square.

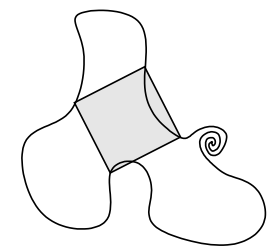

Figure 1. Example for Conjecture 1.

A continuous simple closed curve in the plane is also called a Jordan curve, and it is the same as an injective map from the unit circle into the plane or, equivalently, a topological embedding $S^{1} \hookrightarrow \mathbb{R}^{2}$.

In its full generality Toeplitz's problem is still open. So far it has been solved affirmatively for curves that are "smooth enough" by various authors for varying smoothness conditions; see the next section. All of these proofs are based on the fact that smooth curves inscribe generically an odd number of squares, which can be measured in several topological ways. However, so far none of these methods can be made to work for the general continuous case.

One may think that the general case of the Square Peg Problem can be reduced to the case of smooth curves by approximating a given continuous curve

Benjamin Matschke is a researcher at Max Planck Institute for Mathematics, Bonn. His email address is matschke@ mpim-bonn.mpg.de.

The author was supported by Deutsche Telekom Stiftung, NSF Grant DMS-0635607, an EPDI-fellowship, and MPIM Bonn.

DOI: http://dx.doi.org/10.1090/noti1100

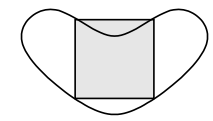

Figure 2. We do not require the square to lie fully inside $\gamma$; otherwise there are counterexamples.

$\gamma$ by a sequence of smooth curves $\gamma_{n}$ : Any $\gamma_{n}$ inscribes a square $Q_{n}$, and by compactness there is a converging subsequence $\left(Q_{n_{k}}\right)_{k}$ whose limit is an inscribed square for the given curve $\gamma$. However, this limit square is possibly degenerate to a point, and so far there is no argument known that can deal with this problem.

Suppose we could show that any smooth (or equivalently any piecewise linear) curve $\gamma$ that contains in its interior a ball of radius $r$ inscribes a square of side length at least $\sqrt{2} r$ (or at least $\varepsilon r$ for some constant $\varepsilon>0$ ). Then the approximation argument would imply that any continuous curve has the same property. However, it seems that we need more geometric than merely topological ideas to show the existence of large inscribed squares.

Other surveys are due to Klee and Wagon [21, Problem 11], Nielsen [30], Denne [4], Karasev [18, 2.6, 4.6], and Pak [32, I.3, I.4]. Jason Cantarella's homepage offers some animations. A Java applet and an extended version of this article are available on my homepage.

In order to raise awareness, let me put 100 euros on each of the Conjectures 1,8 , and 13 . That is, you may earn 300 euros in total.

I want to thank the referees for many very useful comments.

\section{History of the Square Peg Problem}

The Square Peg Problem first appeared in the literature in the conference report [39] in 1911. Toeplitz gave a talk whose second part had the title "On some problems in topology". The report on that second part is rather short: 
b) Ueber einige Aufgaben der Analysis situs. [...]

b) Der Vortragende erzählt von zwei Aufgaben der Analysis Situs, zu denen er gelangt ist, und dann von der folgenden dritten, deren Lösung ihm nur für konvexe Kurven gelungen ist: Auf jeder einfach geschlossenen stetigen Kurve in der Ebene gibt es vier Punkte, welche ein Quadrat bilden. Diskussion: Die Herren Fueter, Speiser, Laemmel, Stäckel, Grossmann.

Here is an English translation:

b) On some problems in topology. [...]

b) The speaker talks about two problems in topology that he obtained, and then about the following third one, whose solution he managed to find only for convex curves: On every simple closed continuous curve in the plane there are four points that form a square. Discussions: Messrs. Fueter, Speiser, Laemmel, Stäckel, Grossmann.

It seems that Toeplitz never published a proof. In 1913 Arnold Emch [6] presented a proof for "smooth enough" convex curves. Two years later Emch [7] published a further proof that requires a weaker smoothness condition. However, he did not note that the special case of smooth convex curves already implies by a limit argument that all convex curves inscribe squares. In a third paper from 1916, Emch [8] proved the Square Peg Problem for curves that are piecewise analytic with only finitely many inflection points and other singularities where the left- and right-side tangents at the finitely many nonsmooth points exist.

Emch states in his second paper [7] that he was not aware of Toeplitz's and his students' work and that the problem was suggested to him by Kempner. From 1906 to 1913 Toeplitz was a postdoc in Göttingen. Aubrey J. Kempner was an English mathematician who finished his Ph.D. with Edmund Landau in Göttingen in 1911. Afterwards he went to the University of Illinois in UrbanaChampaign and stayed there until 1925 according to http://www . maa.org/history/presidents/ kempner.html (another biography of Kempner can be found at http://www. findagrave.com/ cgi-bin/fg. cgi ?page=gr\&GRid=13165695, which claims different dates). Emch joined the faculty of the same university in 1911.

I will let the reader decide whether this is enough information on how all these parts fit together and who considered the Square Peg Problem first. It is usually attributed to Toeplitz.

In 1929 Schnirelman proved the Square Peg Problem for a class of curves that is slightly larger than $C^{2}$. An extended version [37] which also corrects some minor errors was published posthumously in 1944. Guggenheimer [12] states that the extended version still contains errors, which he claims to correct. However, in my point of view, Schnirelman's proof is correct except for some minor errors. His main idea is a bordism argument; below we give some details. Since the transversality machinery was not invented at this time, Schnirelman's proof contains many computations in explicit coordinates. Guggenheimer's main lemma, on the other hand, admits counterexamples; he was not aware that squares can vanish pairwise when one deforms the curve.

Other proofs are due to Hebbert [14] when $\gamma$ is a quadrilateral, Zindler [43] and Christensen [3] for convex curves, Jerrard [16] for analytic curves, Nielsen-Wright [31] for curves that are symmetric across a line or about a point, Stromquist [38] for locally monotone curves, Vrećica-Živaljević [40] for Stromquist's class of curves, Pak [32] for piecewise linear curves, Sagols-Marín [35], [36] for similar discretizations, Cantarella-Denne-McCleary [2] for curves with bounded total curvature without cusps and for $C^{1}$-curves, Makeev [23] for star-shaped $C^{2}$-curves that intersect every circle in at most 4 points (more generally he proved the Circular Quad Peg Problem 9 for such curves, see below), Matschke [26] for a technical open and dense class of curves and for continuous curves in certain bounded domains. In the next section we shall review some of these special cases in more detail.

Pettersson, Tverberg, and Östergård [33] have the latest result, which uses a computer: They showed that any Jordan curve in the $12 \times 12$ square grid inscribes a square whose size is at least $1 / \sqrt{2}$ times the size of the largest axis-parallel square that fits into the interior of the curve.

\section{Special Cases}

Let us discuss some of the above-mentioned proofs in more detail.

\section{Emch's Proof}

Let $\gamma: S^{1} \hookrightarrow \mathbb{R}^{2}$ be the given piecewise analytic curve. Fixing a line $\tau$, Emch considers all secants of $\gamma$ that are parallel to $\tau$ and calls the set of all midpoints of these secants the set of medians $M_{\tau}$. Under some genericity assumptions he proves that for two orthogonal lines $\tau$ and $\tau^{\perp}, M_{\tau}$ intersects $M_{\tau^{\perp}}$ in an odd number of points. Nowadays one could write this down homologically. These intersections correspond to inscribed rhombi, where the two intersecting secants are the two diagonals of the rhombus.

Now he rotates $\tau$ continuously by 90 degrees and argues that $M_{\tau} \cap M_{\tau^{\perp}}$ moves continuously, where at finitely many times two intersection points can merge and disappear or two new intersection 
points can appear. When $\tau$ is rotated by 90 degrees, the one-dimensional family of intersection points closes up to a possibly degenerate union of circle components.

Since $M_{\tau} \cap M_{\tau^{\perp}}$ is odd, Emch argues that an odd number of these components must be $\mathbb{Z} / 4 \mathbb{Z}$ invariant, meaning that if $R_{1} R_{2} R_{3} R_{4}$ is a rhombus in such a component, then $R_{2} R_{3} R_{4} R_{1}$ must also be in the same component. By the mean value theorem, when moving from $R_{1} R_{2} R_{3} R_{4}$ to $R_{2} R_{3} R_{4} R_{1}$ along a component of inscribed rhombi, at some point the diagonals must have equal length. That is, we obtain an inscribed square. This argument also implies that the number of inscribed squares is (generically) odd for Emch's class of curves.

\section{Schnirelman's Proof}

Schnirelman solved the Square Peg Problem for a slightly larger class than $C^{2}$ using an early bordism argument that yields a very conceptual proof. His idea was that the set of inscribed squares can be described as a preimage, for example, in the following way: Let $\gamma: S^{1} \hookrightarrow \mathbb{R}^{2}$ be the given curve. The space $\left(S^{1}\right)^{4}$ parameterizes quadrilaterals that are inscribed in $\gamma$. We construct a so-called test-map,

$$
f_{\gamma}:\left(S^{1}\right)^{4} \rightarrow \mathbb{R}^{6},
$$

which sends a 4-tuple $\left(x_{1}, x_{2}, x_{3}, x_{4}\right)$ of points on the circle to the mutual distances between $\gamma\left(x_{1}\right), \ldots, \gamma\left(x_{4}\right) \in \mathbb{R}^{2}$. Let $V$ be the 2-dimensional linear subspace of $\mathbb{R}^{6}$ that corresponds to the points where all four edges are of equal length and the two diagonals are of equal length. The preimage $f_{\gamma}^{-1}(V)$ is parameterizing the set of inscribed squares, plus a few "degenerate components". The degenerated components consist of points where $x_{1}=x_{2}=x_{3}=x_{4}$-these are the degenerate squares-and more generally of 4-tuples where $x_{1}=x_{3}$ and $x_{2}=x_{4}$.

Now Schnirelman argues as follows: An ellipse inscribes exactly one square up to symmetry. Now deform the ellipse (via some smooth isotopy) into the given curve along other curves $\gamma_{t}, t \in[0,1]$. By smoothness these inscribed squares do not come close to the degenerate quadrilaterals during the deformation; that is, they do not shrink to a point. Thus the nondegenerate part of all preimages $f_{\gamma_{t}}^{-1}(V)$ forms a 1-manifold that connects the solution sets for $\gamma$ and the ellipse, and since 1manifolds always have an even number of boundary points, the parities of the number of inscribed squares on $\gamma$ and on the ellipse coincide.

Thus, any smooth curve inscribes generically an odd number of squares. Here we have swept technical arguments concerning transversality under the rug, which we hope is appreciated by the reader.

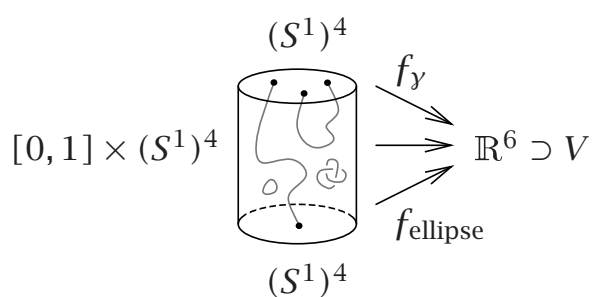

Figure 3. The bordism between the solution sets for $\gamma$ and the ellipse. To simplify the figure we already modded out the symmetry group of the square and omitted the degenerate components.

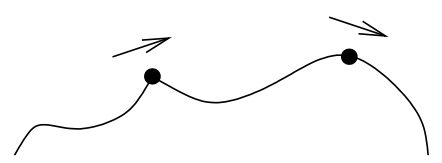

Figure 4. Example of a piece of a locally monotone curve. Note that Figure 1 is not locally monotone because of the spiral.

For general curves, it is difficult to separate the degenerate quadrilaterals in $f^{-1}(V)$ from the squares we are interested in. This is the basic reason why the Square Peg Problem could not be solved completely with the current methods.

\section{Stromquist's Criterion}

Stromquist's class of curves for which he proved the Square Peg Problem is very beautiful, and it is the second strongest one: A curve $\gamma: S^{1} \hookrightarrow \mathbb{R}^{2}$ is called locally monotone if every point $x \in S^{1}$ admits a neighborhood $U$ and a linear functional $\ell: \mathbb{R}^{2} \rightarrow \mathbb{R}$ such that $\left.\ell \circ \gamma\right|_{U}$ is strictly monotone.

Theorem 2 (Stromquist). Any locally monotone embedding $\gamma: S^{1} \hookrightarrow \mathbb{R}^{2}$ inscribes a square.

In his proof Stromquist also considers the set of inscribed rhombi first.

\section{Fenn's Table Theorem}

A beautiful proof for convex curves is due to Fenn [9]. It follows as an immediate corollary from his table theorem.

Theorem 3 (Fenn). Let $f: \mathbb{R}^{2} \rightarrow \mathbb{R}_{\geq 0}$ be a nonnegative function that is zero outside a compact convex disc $D$ and let $a>0$ be an arbitrary real number. Then there exists a square in the plane with side length $a$ and whose center point belongs to $D$ such that $f$ takes the same value on the vertices of the square.

As the reader might guess, Fenn's proof basically uses a mod-2 argument showing that the number of such tables is generically odd.

The table theorem implies the Square Peg Problem for convex curves $\gamma$ by constructing a 


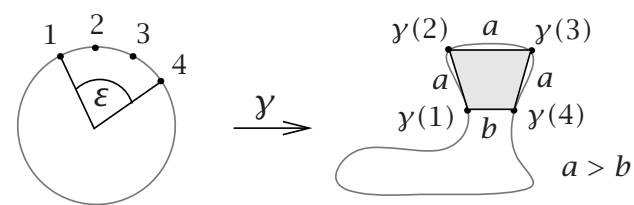

Figure 5. A special trapezoid of size $\varepsilon$.

height function $f: \mathbb{R}^{2} \rightarrow \mathbb{R}_{\geq 0}$ whose level sets $f^{-1}(x)$ are similar to $\gamma$ for all $x>0$.

Zaks [42] found an analogous "chair theorem", where instead of a square table he considers triangular chairs with a fixed direction. KronheimerKronheimer [22] found conditions on $\partial D$ such that the table/chair can be chosen such that all four/three vertices lie in $D$ : namely, $\partial D$ should not inscribe a square/triangle of a smaller size. More table theorems are due to Meyerson [28].

\section{An Open and Dense Criterion}

In [26] the Square Peg Problem was proved for the so far weakest smoothness condition.

Theorem 4. Let $\gamma: S^{1} \hookrightarrow \mathbb{R}^{2}$ be a Jordan curve. Assume that there is $0<\varepsilon<2 \pi$ such that $\gamma$ contains no (or generically an even number of) special trapezoids of size $\varepsilon$. Then $\gamma$ inscribes a square.

Here an inscribed special trapezoid is a 4-tuple of pairwise distinct points $x_{1}, \ldots, x_{4} \in S^{1}$ lying clockwise on $S^{1}$ such that the points $P_{i}:=\gamma\left(x_{i}\right)$ satisfy

||$P_{1}-P_{2}||=|| P_{2}-P_{3}||=|| P_{3}-P_{4}||>|| P_{4}-P_{1}||$

and

$$
|| P_{1}-P_{3} \|=|| P_{2}-P_{4}|| .
$$

The size of this special trapezoid is defined as the length of the clockwise arc in $S^{1}$ from $x_{1}$ to $x_{4}$.

The set of curves without inscribed special trapezoids of a fixed size $\varepsilon$ is open and dense in the space of embeddings $S^{1} \hookrightarrow X$ with respect to the compact-open topology. This theorem is basically the exact criterion that one obtains by applying equivariant obstruction theory to the test-map (1). Vrećica and Živaljević [40] are the first to apply obstruction theory to the Square Peg Problem, and they proved it for Stromquist's class of locally monotone curves.

\section{An Explicit Open Criterion}

All previous criteria on curves for which the Square Peg Problem was proved are defined by local smoothness conditions. The following criterion from [26] is a global one which yields an open set of not necessarily injective curves in $C^{0}\left(S^{1}, \mathbb{R}^{2}\right)$ with respect to the $C^{0}$-topology or, equivalently, the compact-open topology.

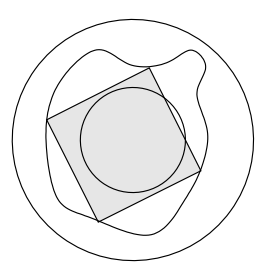

Figure 6. Example for Theorem 5.

Theorem 5. Let $A$ denote the annulus $\left\{x \in \mathbb{R}^{2} \mid 1 \leq\right.$ $\|x\| \leq 1+\sqrt{2}\}$. Suppose that $\gamma: S^{1} \rightarrow A$ is a continuous closed curve in $A$ that is nonzero in $\pi_{1}(A)=\mathbb{Z}$. Then $\gamma$ inscribes a square of side length at least $\sqrt{2}$.

It is open whether the outer radius $1+\sqrt{2}$ of $A$ can be increased by some small $\varepsilon>0$.

The proof idea is very simple: If the annulus $A$ is thin enough, then the set of squares with all vertices in $A$ splits into two connected components: big squares and small squares. A generic curve that represents a generator of $\pi_{1}(A)$ inscribes an odd number of big squares (and an even number of small squares).

\section{Related Problems}

\section{The Number of Inscribed Squares}

Popvassilev [34] constructed for any $n \geq 1$ a smooth convex curve that has exactly $n$ inscribed squares, every square being counted exactly once and not with multiplicity. All but one of the $n$ squares in his construction are nongeneric. They will disappear immediately after deforming the curve by a suitable $C^{\infty}$-isotopy.

In [26] this author gave the parity of the number of squares on generic smooth immersed curves in the plane, which depends not only on the isotopy type of the immersion but also on the intersection angles.

Van Heijst proves in his upcoming master's thesis [15] that any real algebraic curve in $\mathbb{R}^{2}$ of degree $d$ inscribes either at most $\left(d^{4}-5 d^{2}+4 d\right) / 4$ or infinitely many squares. For this he makes use of Bernstein's theorem, which states that the number of common zeros in $\left(\mathbb{C}^{*}\right)^{k}$ of $k$ generic Laurent polynomials in $k$ variables with prescribed Newton polytopes equals the mixed volume of these polytopes.

\section{Inscribed Triangles}

It is not hard to show that any smooth embedding $\gamma: S^{1} \rightarrow \mathbb{R}^{2}$ inscribes arbitrary triangles, even if we prescribe where one of the vertices has to sit. Moreover, the set of all such inscribed triangles determines a homology class $\alpha \in H_{1}\left(P_{3}, \mathbb{Z}\right)=\mathbb{Z}$, where $P_{3}$ is the set of 3 -tuples of points on $\gamma$ that lie counterclockwise on the curve. The class 


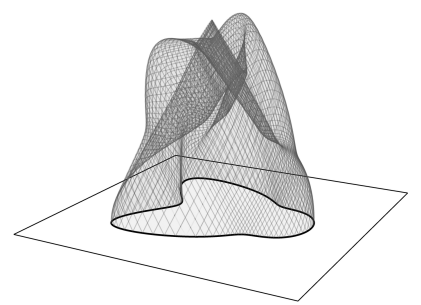

Figure 7. The image of $f$, a self-intersecting Möbius strip with boundary $\gamma$.

$\alpha$ turns out to be a generator, as one sees from inspecting the situation for the circle.

For continuous curves Nielsen [29] proved the following version of the result:

Theorem 6 (Nielsen). Let $T$ be an arbitrary triangle and $\gamma: S^{1} \rightarrow \mathbb{R}^{2}$ an embedded circle. Then there are infinitely many triangles inscribed in $\gamma$ which are similar to $T$, and if one fixes a vertex of smallest angle in $T$, then the set of the corresponding vertices on $\gamma$ is dense in $\gamma$.

\section{Inscribed Rectangles}

Instead of squares one may ask whether any embedded circle in the plane inscribes a rectangle. If one does not prescribe the aspect ratio, then the answer is affirmative.

Theorem 7 (Vaughan). Any continuous embedding $\gamma: S^{1} \hookrightarrow \mathbb{R}^{2}$ inscribes a rectangle.

Vaughan's proof, which appeared in Meyerson [28], is very beautiful: $\mathbb{Z}_{2}:=\mathbb{Z} / 2 \mathbb{Z}$ acts on the torus $\left(S^{1}\right)^{2}$ by permuting the coordinates, and the quotient space $\left(S^{1}\right)^{2} / \mathbb{Z}_{2}$ is a Möbius strip. The proof of Theorem 7 uses the fact that the map $f:\left(S^{1}\right)^{2} / \mathbb{Z}_{2} \rightarrow \mathbb{R}^{2} \times \mathbb{R}_{\geq 0}$ given by

$$
f(x, y)=((\gamma(x)+\gamma(y)) / 2,\|\gamma(x)-\gamma(y)\|)
$$

must have a double point; otherwise it would extend to an embedding of $\mathbb{R} P^{2}$ into $\mathbb{R}^{3}$ by gluing to that Möbius strip the disc $I \times\{0\}$, where $I \subset \mathbb{R}^{2}$ is the interior of $\gamma$. The double point corresponds to two secants of $\gamma$ having the same length and the same midpoint. Hence this forms an inscribed rectangle.

If we furthermore prescribe the aspect ratio of the rectangle, then the problem is wide open, even for smooth or piecewise linear curves.

Conjecture 8 (Rectangular Peg Problem). Every $C^{\infty}$ embedding $\gamma: S^{1} \rightarrow \mathbb{R}^{2}$ contains four points that are the vertices of a rectangle with a prescribed aspect ratio $r>0$.

This conjecture is highly interesting, since the standard topological approach does not yield a proof: The equivariant homology class of the solution set, a $\mathbb{Z}$-valued smooth isotopy invariant of the curve, turns out to be zero. For example, an ellipse inscribes a positive and a negative rectangle. Stronger topological tools fail as well. It seems again that more geometric ideas are needed.

Equivalently we could state Conjecture 8 for all piecewise linear curves. Proofs exist only for the case $r=1$, which is the smooth Square Peg Problem, for arbitrary $r$ in case the curve is close to an ellipse, see Makeev [23] and Conjecture 9 below; and for $r=\sqrt{3}$ in case the curve is close to convex, see [26].

A proof for the Rectangular Peg Problem was claimed by Griffiths [10], but it contains errors regarding the orientations. Essentially, he calculated that the number of inscribed rectangles of the given aspect ratio counted with appropriate signs and modulo symmetry is 2 . However, zero is correct.

\section{Other Inscribed Quadrilaterals}

It is natural to ask what other quadrilaterals can be inscribed into closed curves in the plane. Since the unit circle is a curve, those quadrilaterals must be circular; that is, they must have a circumcircle.

Depending on the class of curves that we look at, the following two conjectures seem reasonable.

Conjecture 9 (Circular Quad Peg Problem). Let $Q$ be a circular quadrilateral. Then any $C^{\infty}$ embedding $\gamma: S^{1} \rightarrow \mathbb{R}^{2}$ admits an orientation-preserving similarity transformation that maps the vertices of $Q$ into $\gamma$.

Makeev [23] proved a first instance of this conjecture, namely, for the case of star-shaped $C^{2}$-curves that intersect every circle in at most 4 points.

Furthermore, Karasev [20] proved that, for any smooth curve and a given $Q=A B C D$, either this conjecture holds or one can find two inscribed triangles similar to $A B C$ such that the two corresponding fourth vertices $D$ coincide (but $D$ may not lie on $\gamma$ ). The proof idea is a beautiful geometric volume argument. It should be stressed that most open problems discussed here are geometric problems rather than topological ones: We understand the basic algebraic topology here quite well but not the restrictions on the topology that the geometry dictates. New geometric ideas such as Karasev's are needed.

Conjecture 10 (Trapezoidal Peg Problem). Let T be an isosceles trapezoid. Then any piecewise-linear embedding $\gamma: S^{1} \rightarrow \mathbb{R}^{2}$ inscribes a quadrilateral similar to $T$.

The reason for restricting the latter conjecture to isosceles trapezoids, that is, trapezoids with circumcircle, is that all other circular quadrilaterals cannot be inscribed into very thin triangles. This was observed by Pak [32]. 


\section{Other Inscribed Polygons}

For any $n$-gon $P$ with $n \geq 5$ it is easy to find many curves that do not inscribe $P$. If we do not require all vertices to lie on $\gamma$, then Makeev has some results for circular pentagons; see [25].

Alternatively, we can relax the angle conditions; that is, we require only that the edge ratios are the same as the ones in a given polygon $P$. Then as for the triangles above, one can show that the set of such $n$-gons represents the generator of $H_{1}\left(P_{n} ; \mathbb{Z}\right)=\mathbb{Z}$, where $P_{n}$ is the set of $n$-tuples of points on $\gamma$ that lie counterclockwise on the curve; see Meyerson [27], Wu [41], Makeev [25], and Vrećica-Živaljević [40].

\section{Higher Dimensions}

In higher dimensions one may ask whether any $(n-1)$-sphere that is smoothly embedded in $\mathbb{R}^{n}$ inscribes an $n$-cube in the sense that all vertices of the cube lie on the sphere. However, most smooth embeddings $S^{n-1} \hookrightarrow \mathbb{R}^{n}$ do not inscribe an $n$-cube for $n \geq 3$, in the sense that these embeddings form an open and dense subset of all smooth embeddings in the compact-open topology, a heuristic reason being that the number of equations to fulfill is larger than the degrees of freedom. An explicit example is the boundaries of very thin simplices, as was noted by Kakutani [17] for $n=3$. Hausel-Makai-Szúcs [13] proved that the boundary of any centrally symmetric convex body in $\mathbb{R}^{3}$ inscribes a 3-cube.

If we do not want to require further symmetry on the embedding $S^{n-1} \hookrightarrow \mathbb{R}^{n}$, then crosspolytopes are more suitable higher analogs of squares: The regular $n$-dimensional crosspolytope is the convex hull of $\left\{ \pm e_{i}\right\}$ where $e_{i}$ are the standard basis vectors in $\mathbb{R}^{n}$.

Theorem 11 (Makeev, Karasev). Let $n$ be an odd prime power. Then every smooth embedding $\Gamma$ : $S^{n-1} \rightarrow \mathbb{R}^{n}$ contains the vertices of a regular $n$ dimensional crosspolytope.

The $n=3$ case was posed as Problem 11.5 in Klee and Wagon [21]. This was answered affirmatively by Makeev [24]. Karasev [19] generalized the proof to arbitrary odd prime powers. Akopyan and Karasev [1] proved the same theorem for $n=3$ in case $\Gamma$ is the boundary of a simple polytope by a careful and nontrivial limit argument from the smooth case.

Gromov [11] proved a similar theorem for inscribed simplices.

Theorem 12 (Gromov). Any compact set $S \subset \mathbb{R}^{d}$ with $C^{1}$-boundary and nonzero Euler characteristic inscribes an arbitrary given simplex up to similarity on its boundary $\partial S$.

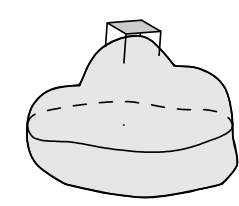

Figure 8. Intuition behind Conjecture 13: Think of a square table for which we want to find a spot on Earth such that all four table legs are at the same height.

Let us finish with the following table problem on the sphere.

Conjecture 13 (Table problem on $S^{2}$ ). Suppose $x_{1}, \ldots, x_{4} \in S^{2} \subset \mathbb{R}^{3}$ are the vertices of a square that is inscribed in the standard 2-sphere, and let $h: S^{2} \rightarrow \mathbb{R}$ be a smooth function. Then there exists a rotation $\rho \in S O(3)$ such that $h\left(\rho\left(x_{1}\right)\right)=\cdots=$ $h\left(\rho\left(x_{4}\right)\right)$.

So far this result has been proven only when $x_{1}, \ldots, x_{4}$ lie on a great circle (see Dyson [5]), since this is the only case in which the generic number of solutions is odd. The critical points of $h$ can be thought of as the spots on which you can put an infinitesimally small table.

\section{References}

1. ARSENIY AKOPYAN and ROMAN N. KARASEV, Inscribing a regular octahedron into polytopes, Discrete Math. 313 (2013), no. 1, 122-128.

2. JASOn CANTARElla, Elizabeth Denne, and JOHn MCCLEARY, Transversality in configuration spaces and the Square Peg Problem, in preparation.

3. CARL MARIUS Christensen, A square inscribed in a convex figure (in Danish), Matematisk Tidsskrift B 1950 (1950), 22-26.

4. ElizABETH DENNE, Inscribed squares: Denne speaks, http://quomodocumque.wordpress.com/2007/08/ 31/inscribed-squares-denne-speaks, 2007, Guest post on Jordan S. Ellenberg's blog Quomodocumque.

5. FreEMAN J. Dyson, Continuous functions defined on spheres, Ann. Math. 54 (1951), no. 2, 534-536.

6. ARNOLD EMch, Some properties of closed convex curves in a plane, Amer. J. Math. 35 (1913), 407-412.

7. Amer. J. Math. 37 (1915), 19-28.

8. __ On some properties of the medians of closed continuous curves formed by analytic arcs, Amer. $J$. Math. 38 (1916), no. 1, 6-18.

9. Roger FEnN, The table theorem, Bull. London Math. Soc. 2 (1970), 73-76.

10. H. BRIAN GRIFFITHS, The topology of square pegs in round holes, Proc. London Math. Soc. 62 (1990), no. 3, 647-672.

11. Mikhail L. Gromov, Simplexes inscribed on a hypersurface (Russian), Matematicheskie Zametki 5 (1969), 81-89.

12. HeInRICH W. GugGENHEIMER, Finite sets on curves and surfaces, Israel J. Math. 3 (1965), 104-112.

13. TAMÁS HAUSEL, ENDRE MAKAI JR., and ANDRÁS SZÜCS, Inscribing cubes and covering by rhombic dodecahedra 
via equivariant topology, Mathematika 47 (2002), no. 12, 371-397.

14. Clarence M. Hebbert, The inscribed and circumscribed squares of a quadrilateral and their significance in kinematic geometry, Ann. of Math. (2) 16 (1914/15), no. $1-4,38-42$.

15. WOUTER VAN HEIJST, master's thesis, in preparation.

16. RICHARD P. JERRARD, Inscribed squares in plane curves, Trans. Amer. Math. Soc. 98 (1961), 234-241.

17. SHIzUO KAKUTANI, A proof that there exists a circumscribing cube around any bounded closed convex set in $\mathbb{R}^{3}$, Ann. Math. 43 (1942), no. 4, 739-741.

18. ROMAN N. KARASEV, Topological methods in combinatorial geometry, Russian Math. Surveys 63 (2008), no. $6,1031-1078$.

19. Inscribing a regular crosspolytope, http:// arxiv.org/abs/0905.2671, 2009.

20. Roman N. KARASEV and Aleksei Yu. VoloviKov, A note on Makeev's conjectures, http://arxiv. org/abs/1002.4070, 2010.

21. Victor KLEE and StAn WAgon, Old and New Unsolved Problems in Plane Geometry and Number Theory, Dolciani Mathematical Expositions, Math. Assoc. America, 1996.

22. Erwin H. Kronheimer and Peter B. Kronheimer, The tripos problem, J. London Math. Soc. (2) 24 (1981), no. 1, 182-192.

23. VLADIMIR V. MAKEEV, On quadrangles inscribed in a closed curve, Math. Notes 57 (1995), no. 1-2, 91-93.

$24 . \ldots$ Universally inscribed and outscribed polytopes, Ph.D. thesis, Saint-Petersburg State University, 2003.

25. $\ldots$, On quadrangles inscribed in a closed curve and the vertices of the curve, J. Math. Sci. 131 (2005), no. 1, 5395-5400.

26. BENJAMIN MATSCHKE, Equivariant topology methods in discrete geometry, Ph.D. thesis, Freie Universität Berlin, 2011.

27. MARK D. MEYerson, Equilateral triangles and continuous curves, Polska Akademia Nauk. Fundamenta Mathematicae 110 (1980), no. 1, 1-9.

28. $\_$, Balancing acts, Topology Proc. 6 (1981), no. 1, 59-75.

29. MARK J. NielSen, Triangles inscribed in simple closed curves, Geometriae Dedicata 43 (1992), 291-297.

30. Web page on Figures Inscribed in Curves, http://www.webpages.uidaho.edu/ markn/squares/ 2000.

31. MARK J. NiELSEN and STEPHEN E. Wright, Rectangles inscribed in symmetric continua, Geom. Dedicata 56 (1995), no. 3, 285-297.

32. IGOR PAK, Lectures on Discrete and Polyhedral Geometry, http://math.ucla.edu/ pak/book.htm 2010.

33. Ville H. Pettersson, Helge TVerberg, and PAtric R. J. ÖSTERGÅRD, A note on Toeplitz' square problem, submitted, 2013.

34. Strashimir G. Popvassilev, On the number of inscribed squares of a simple closed curve in the plane, http://arxiv.org/abs/0810.4806, 2008.

35. FELIÚ SAGOLS and RAÚL MARÍn, The inscribed square conjecture in the digital plane, Combinatorial Image Analysis, Lecture Notes in Comput. Sci., vol. 5852, Springer, 2009, pp. 411-424.

36. _ Two discrete versions of the inscribed square conjecture and some related problems, Theoret. Comput. Sci. 412 (2011), no. 15, 1301-1312.
37. LEV G. SCHNIRELMAN, On some geometric properties of closed curves (in Russian), Usp. Mat. Nauk 10 (1944), 34-44. Available at http://ega-math. narod.ru/Nquant/Square.djv. Posthumous reproduction and extension of the author's original article in Sbornik Rabot Matematičeskogo Razdela Sekcii Estestvennyh i Točnyh Nauk Komakademii, Moscow, 1929.

38. WALTER R. STROMQUIST, Inscribed squares and squarelike quadrilaterals in closed curves, Mathematika 36 (1989), 187-197.

39. Отто Toeplitz, Ueber einige Aufgaben der Analysis situs, Verhandlungen der Schweizerischen Naturforschenden Gesellschaft in Solothurn 4 (1911), 197.

40. Siniša VRećicA and RAde T. Živaljević, FultonMacPherson compactification, cyclohedra, and the polygonal pegs problem, Israel J. Math. 184 (2011), no. 1, 221-249.

41. YING-QING WU, Inscribing smooth knots with regular polygons, Bull. London Math. Soc. 36 (2004), no. 2, 176 180.

42. JoSEPH ZAKS, The chair theorem, Proceedings of the Second Louisiana Conference on Combinatorics, Graph Theory and Computing (Baton Rouge, La.), Louisiana State Univ., 1971, pp. 557-562.

43. KONRAD ZINDLER, Über konvexe Gebilde, Monatshefte für Mathematik und Physik 31 (1921), 25-56. 